INGENIERÍA CIVIL

\title{
Influencia del origen y la mineralogía de las arenas en la resistencia a licuación
}

CIVIL ENGINEERING

\section{Influence of sand origin and mineralogy on liquefaction resistance}

\author{
Eimar A. Sandoval ${ }^{\star}$, Miguel A. Pando*** \\ * Escuela de Ingeniería Civil y Geomática, Universidad del Valle \\ ** Department of Civil and Environmental Engineering, University of North Carolina at Charlotte \\ §eimar.sandoval@correounivalle.edu.co,mpando@uncc.edu
}

(Recibido: Marzo 30 de 2011 -Aceptado: Mayo 15 de 2012 -Versión Final: Junio 22 de 2012)

\begin{abstract}
Resumen
Los métodos disponibles en la literatura para evaluar la resistencia a licuación de arenas se basan principalmente en investigaciones sobre arenas de origen terrígeno con mineralogía de sílice o cuarzo. Esto es debido en parte a que las arenas terrígenas son las más abundantes. Sin embargo existen otros tipos de arenas en términos de origen, mineralogía y forma de sus granos, cuya susceptibilidad a licuación no ha sido estudiada en mayor detalle. Este artículo busca contribuir a llenar este vacío, al presentar resultados de un programa experimental detallado para determinar la resistencia a licuación de arenas calcáreas no cementadas. El estudio involucró caracterización mineralógica, determinación de propiedades índice principales, línea de estado crítico y ensayos triaxiales cíclicos no drenados con consolidación isotrópica, sobre una arena calcárea no cementada. Con fines de comparación, se realizaron pruebas similares sobre la arena de sílice estándar de Ottawa. Los resultados mostraron que las arenas calcáreas tuvieron mayor resistencia a licuación que las arenas de sílice, cuando fueron probadas bajo condiciones similares. También se encontraron diferencias importantes en la generación de presiones de poros y en la acumulación de deformaciones axiales durante la fase de carga cíclica en los ensayos triaxiales.
\end{abstract}

Palabras Claves: Arenas calcáreas, arenas terrígenas, arena no cementada, organismos biogénicos, resistencia a licuación, presiones de poros, deformación axial.

\begin{abstract}
The available methods in the literature to assess liquefaction resistance of sands are based on studies using terrigenous sands with silica or quartzitic mineralogy. This is in part because terrigenous sands are the most abundant. However, there are other kinds of sands, in terms of origin, mineralogy and grain shape, for which their liquefaction susceptibility has not been studied in enough detail. This paper tries to fill this knowledge gap by presenting results of a detailed experimental program carried out to determine the liquefaction resistance of uncemented calcareous sand. This study involved mineralogical characterization, main index properties determination, critical state line, and undrained cyclic triaxial tests with isotropic consolidation on the calcareous sand. For comparison purposes, similar tests were performed on Ottawa standard silica sand. The results showed that the calcareous sands exhibited higher liquefaction resistance than silica sand, when tested under similar test conditions. Significant differences were also observed in terms of pore pressure generation and accumulation of axial strain during the undrained cyclic loading phase of the triaxial tests.
\end{abstract}

Keywords: Calcareous sands, terrigenous sands, uncemented sand, biogenic organisms, liquefaction resistance, pore pressures, axial strain. 


\section{Introducción}

El fenómeno de licuación es un serio problema en la ingeniería geotécnica, el cual se evidencia después de terremotos fuertes o ante vibraciones. La licuación suele presentarse generalmente en suelos arenosos, aunque algunas investigaciones han concluido que suelos con finos no plásticos podrían ser susceptibles de licuarse (Polito \& Martin, 2001, Carraro et al., 2003). En esta investigación se consideró como licuación la pérdida en la resistencia al corte del suelo como consecuencia del incremento en las presiones de poros durante el terremoto o vibración, las cuales pueden aumentar considerablemente, hasta el punto que el esfuerzo efectivo de la muestra llega a cero o casi cero. Esta definición de licuación suele referirse como licuación cíclica (Robertson \& Wride, 1997), y puede ocurrir tanto en suelos con comportamiento contráctil como dilatante. Licuación cíclica ocurre en suelos donde los esfuerzos cortantes iniciales no son significativos en comparación con los esfuerzos cíclicos inducidos por el terremoto o vibración. Bajo esta condición la carga cíclica se caracteriza por esfuerzos cortantes que oscilan de positivos a negativos ("shear stress reversal"), y sí la duración y magnitud de la carga cíclica es suficiente, se llega a la condición de licuación cíclica representada por esfuerzo efectivo de confinamiento igual a cero. Cabe anotar que esta definición no es equivalente a licuación de flujo ("flow liquefaction") donde la licuación puede ocurrir bajo carga cíclica o monotónica y se caracteriza por la pérdida repentina de resistencia al corte hasta llegar al estado crítico (Robertson \& Wride, 1997).

Ocurrencia de licuación de suelos suele reportarse en la mayoría de terremotos de gran magnitud y normalmente se manifiesta en forma de volcanes de eyección ("sand boils"), daño o colapso de estructuras por asentamientos excesivos o falla de capacidad portante, grandes movimientos laterales de bloque, fallas de taludes, entre otras. A pesar de los múltiples avances relacionados al entendimiento del fenómeno de licuación aún queda mucho por investigar. Por ejemplo, debido a que la mayoría de las investigaciones y experiencias recopiladas sobre resistencia a licuación están basadas en observaciones sobre arenas terrígenas de sílice o cuarzo, es importante estudiar la influencia que tiene la mineralogía de las arenas en la resistencia a licuación. Relacionado a esta necesidad, este artículo presenta resultados de una investigación que ilustra la importancia de considerar origen y mineralogía en el análisis de licuación cíclica. Específicamente se presenta el resumen de una investigación experimental llevada a cabo sobre una arena calcárea no cementada extraída de una playa en Cabo Rojo, Puerto Rico. El programa involucró caracterización mineralógica, línea de estado crítico, determinación de propiedades índice principales y ensayos triaxiales cíclicos no drenados sobre muestras reconstituidas de arena calcárea consolidadas isotrópicamente. En los ensayos triaxiales cíclicos se utilizaron tres niveles de esfuerzo efectivo de consolidación y cuatro densidades relativas. Con fines de comparación, análisis mineralógico, determinación de propiedades índice y ensayos triaxiales cíclicos fueron realizados sobre la arena de sílice estándar de Ottawa, bajo algunas densidades relativas y esfuerzos efectivos de consolidación similares a los utilizados para la arena calcárea.

\section{Metodología}

\subsection{Programa experimental}

El programa experimental incluyó la determinación del contenido de carbonato de calcio mediante reacción con ácido clorhídrico $(\mathrm{HCl})$, micrografías mediante microscopio de barrido electrónico, determinación de propiedades índice, línea de estado crítico y ensayos triaxiales cíclicos no drenados sobre muestras reconstituidas consolidadas isotrópicamente. Se realizaron en total 39 ensayos triaxiales cíclicos; de los cuales, 31 fueron ejecutados sobre la arena calcárea de Cabo Rojo y 8 sobre la arena de sílice Ottawa.

\subsection{Descripción de las arenas ensayadas}

Las arenas calcáreas fueron extraídas de la Playa Puerto Real en Cabo Rojo al suroeste de Puerto Rico, son de color habano a amarillo claro, 
pobremente gradadas, con granos sub-angulares a angulares, de tamaño fino a medio, alta porosidad interna y alto contenido de carbonato de calcio $\mathrm{CaCO}_{3}$ (mayor al 91\%). La Figura 1 ilustra micrografías de las arenas calcáreas de Cabo Rojo, en las cuales se pueden observar algunos de sus componentes biogénicos, su alta porosidad interna, su alta rugosidad, entre otras propiedades específicas. Las arenas silíceas de Ottawa son de color blanco, pobremente gradadas con tamaños entre el tamiz No $20(0.841 \mathrm{~mm})$ y el tamiz No 30 $(0.595 \mathrm{~mm})$, con granos finos sólidos $\mathrm{y}$ redondeados, y contenido de $\mathrm{CaCO}_{3}$ menor al $1 \%$.

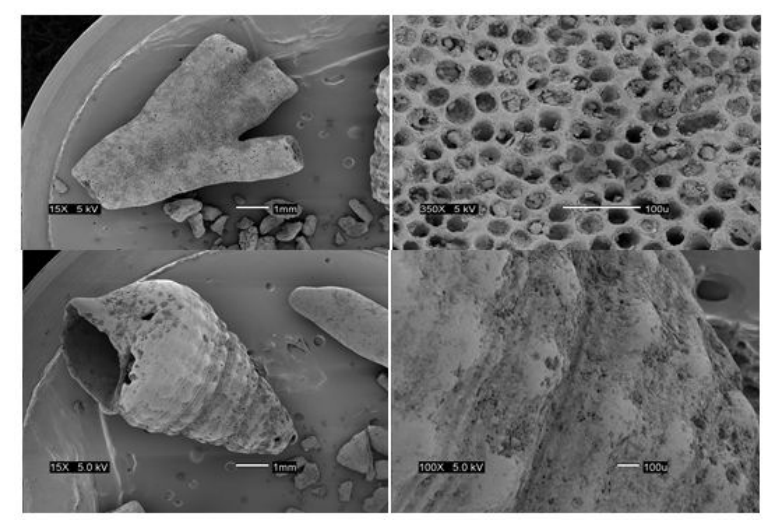

Figura 1. Micrografías de las arenas calcáreas de Cabo Rojo (Tomada de Cataño \& Pando, 2010)

La Tabla 1 resume las principales propiedades índice de las dos arenas usadas, donde se pueden observar importantes diferencias tanto en su composición mineralógica como en las propiedades de los granos. Por ejemplo, debido a la composición mineralógica de las arenas calcáreas, estas arenas presentan una alta gravedad específica del orden de 2.84, la cual es mayor que el valor típico de 2.65 de las arenas de sílice. A causa de su porosidad interna, las arenas calcáreas presentaron grandes relaciones de vacíos $(e)$, entre 1.51 y 2.07, mientras que las arenas de sílice tuvieron valores de $e$ entre 0.50 y 0.78 . La Figura 2 ilustra las curvas de distribución granulométrica para ambas arenas. En esta figura también se observan algunas diferencias en el tamaño máximo y rangos de tamaños para las dos arenas estudiadas.

El comportamiento esfuerzo-deformación de estas arenas calcáreas fue previamente estudiado por
Cataño \& Pando (2010), quienes encontraron ángulos de fricción interna efectiva pico $\left(\phi^{\prime}\right)$ de $39.8^{\circ}$ y $41.2^{\circ}$, para estados medio $\left(\mathrm{D}_{\mathrm{r}} \approx 44 \%\right)$ y denso $\left(\mathrm{D}_{\mathrm{r}} \approx 95 \%\right)$, respectivamente.

Tabla 1. Principales propiedades índice de las arenas usadas

\begin{tabular}{cccc}
\hline Parámetro & $\begin{array}{c}\text { Arena calcárea Cabo } \\
\text { Rojo }\end{array}$ & $\begin{array}{c}\text { Arena de sílice } \\
\text { Ottawa }\end{array}$ & $\begin{array}{c}\text { NORMA } \\
\text { TÉCNICA }\end{array}$ \\
\hline $\mathrm{D}_{10}(\mathrm{~mm})$ & 0.24 & 0.65 & \\
$\mathrm{D}_{50}(\mathrm{~mm})$ & 0.37 & 0.75 & ASTM \\
$\mathrm{C}_{\mathrm{u}}$ & 1.75 & 2.1 & D422-63 (2002) \\
$\mathrm{C}_{\mathrm{c}}$ & 0.94 & 1.1 & \\
$\mathrm{G}_{\mathrm{s}}$ & 2.84 & 2.65 & ASTM \\
& & & D854-06 \\
$\gamma_{\min }\left(\mathrm{KN} / \mathrm{m}^{3}\right)$ & 9.1 & 14.6 & ASTM \\
$\mathrm{e}_{\max }$ & 2.07 & 0.78 & D4254-00 \\
$\gamma_{\max }\left(\mathrm{KN} / \mathrm{m}^{3}\right)$ & 11.1 & 17.3 & Alternative \\
$\mathrm{e}_{\min }$ & 1.51 & 0.50 & Method \\
\hline \multicolumn{2}{c}{}
\end{tabular}

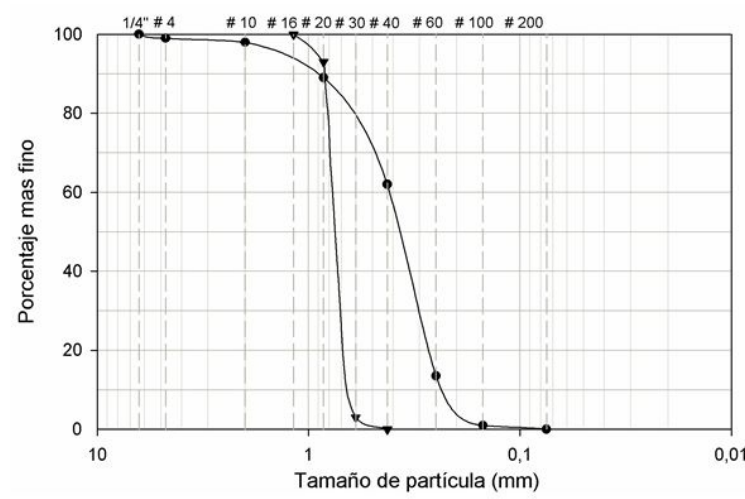

Figura 2. Distribución granulométrica de las arenas ensayadas. Arena calcárea de Cabo Rojo, $\nabla$ Arena silícea de Ottawa

\subsection{Línea de estado crítico}

Dentro del marco de la mecánica de suelos de estado crítico, el comportamiento de una arena bajo cargas externas puede ser contráctil o dilatante, según sea su estado inicial con respecto a la línea de estado crítico (LEC). El estado inicial de una muestra de suelo suele definirse, para fines de licuación, en términos de su relación de vacíos (e) y su estado de esfuerzos $\left(\sigma_{3}^{\prime} \circ p^{\prime}\right)$. Para el análisis de licuación, las muestras que se encuentran por encima o a la derecha de la LEC tienen un comportamiento contráctil, donde el esqueleto tiende a comprimirse, y de esta forma 
son más susceptibles a experimentar licuación. Entre más alejada hacia la derecha o hacia arriba de la LEC se encuentre una muestra, mayor será su potencial contractivo y su susceptibilidad a la licuación. Por otra parte, muestras con estado inicial por debajo o a la izquierda de la LEC se refieren a arenas en estado dilatante, y entre más alejada hacia abajo o hacia la izquierda de la LEC se encuentre una muestra, menor será su potencial de licuación. En la Sección 3.1 se presentan más detalles de estas tendencias.

La posición de la línea de estado crítico (LEC) fue definida usando resultados experimentales de Cataño \& Pando (2010) y los de esta investigación. La Figura 3 muestra los diversos puntos experimentales utilizados para definir LEC, representando varias relaciones de vacío iniciales de muestras que alcanzaron el estado crítico. En esta gráfica, los puntos representados por círculos con relleno fueron obtenidos mediante ensayos triaxiales monotónicos consolidados isotrópicamente y bajo corte no drenado (triaxiales CIU) reportados por Cataño \& Pando (2010). Los puntos representados por círculos sin relleno fueron obtenidos en esta investigación usando el método simplificado propuesto por Santamarina \& Cho (2001).

La Figura 4 ilustra la LEC inferida de los datos presentados en la Figura 3. Nótese que en esta figura, definida en el espacio e $-\sigma_{3}^{\prime}$, la LEC se representa por dos rectas. Esto no es atípico, y concuerda con resultados presentados para otras arenas, donde la LEC es una curva cóncava hacia abajo. (Ver por ejemplo resultados para la arena de Toyoura reportados por Ishihara, 1996).

LaFigura 4 muestra también la posición promedio de los ocho grupos de muestras de las arenas calcáreas de Cabo Rojo con respecto a la LEC. Cada punto representa el estado promedio de las muestras después de la consolidación, justo antes de iniciarse la fase de carga cíclica no drenada. Cada grupo corresponde a un mismo esfuerzo efectivo de consolidación y densidad relativa. En la Figura 4 puede notarse que seis de los ocho grupos de muestras se ubicaron por encima de la LEC, es decir corresponden a comportamiento contráctil.

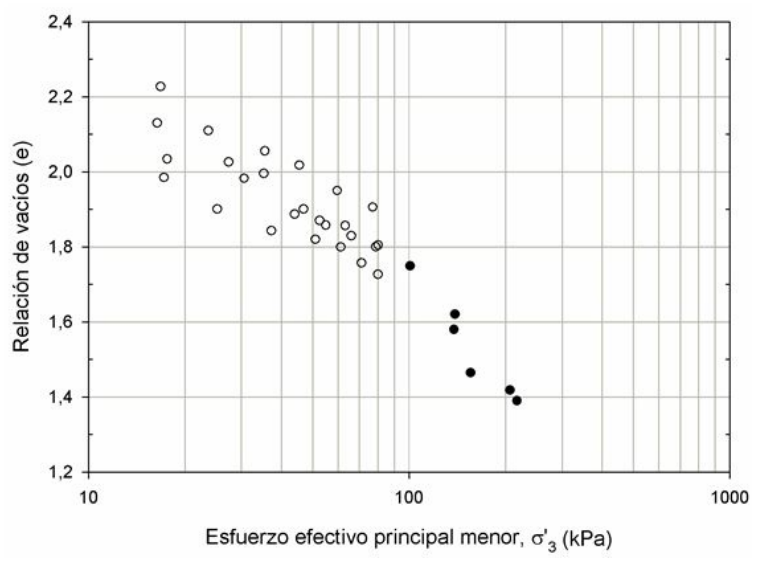

Figura 3. Linea de estado crítico para la arena calcárea de Cabo Rojo. - Cataño \& Pando, 2010, o Método Santamarina \& Cho, 2001

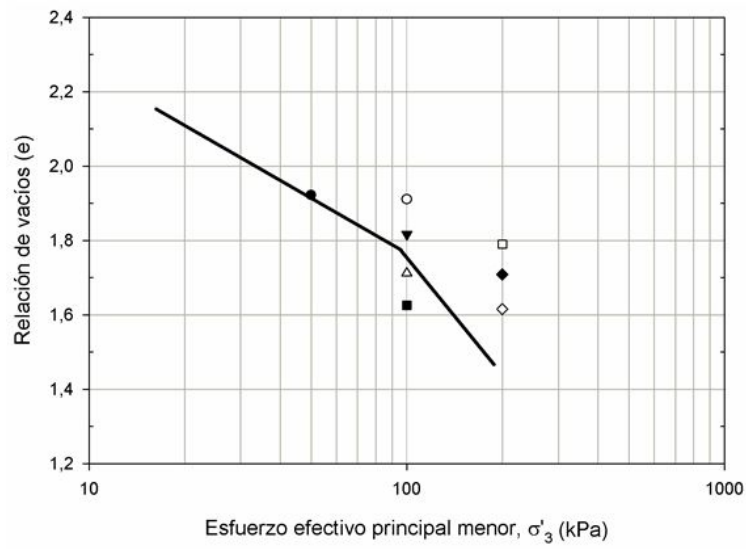

Figura 4. Posición de las muestras de la arena calcárea de Cabo Rojo con respecto a la línea de estado crítico, antes de iniciar la fase cíclica. $-\sigma_{3 c o n}^{\prime}=50 \mathrm{kPa} D_{r}=26 \%, 0 \sigma_{3 c o n}^{\prime}=$ $100 \mathrm{kPa} D_{r}=27 \%, \nabla \sigma_{3 c o n}^{\prime}=100 \mathrm{kPa} D_{r}=45 \%, \Delta \sigma_{3 c o n}^{\prime}=100$ $k P a D_{r}=64 \%, \square \sigma_{3 c o n}^{\prime}=100 \mathrm{kPaD} D_{r}=80 \%, \square \sigma_{3 c o n}^{\prime}=200 \mathrm{kPa}$ $D_{r}=50 \%, \diamond \sigma_{3 c o n}^{\prime}=200 \mathrm{kPa} D_{r}=65 \%, \diamond \sigma_{3 c o n}^{\prime}=200 \mathrm{kPaD} D_{r}=$ $82 \%$,

\subsection{Procedimiento de ensayos triaxiales cíclicos}

La resistencia a licuación de las dos arenas objeto de esta investigación fue evaluada mediante ensayos triaxiales cíclicos no drenados, según la metodología de la Norma ASTM-D5311. En promedio, las muestras para los ensayos triaxiales cíclicos tuvieron una altura y un diámetro de 101.6 $\mathrm{mm}$ y $50.8 \mathrm{~mm}$ respectivamente. Todos los especímenes se prepararon usando el método de apisonamiento húmedo ("moist tamping"). Este 
método consiste en agregar cierta cantidad de agua y compactar las muestras mediante un pisón, dentro de un cilindro metálico recubierto con una membrana de látex y sometido a vacío para garantizar la forma y consistencia de la muestra. El suelo se colocó en cinco capas usando el procedimiento de sub-compactación sugerido por Ladd (1978). Este método recomienda que la densidad inicial de las capas inferiores sea menor, debido a que el peso de las capas superiores incrementará su densidad al final de la preparación de la muestra. El apisonamiento húmedo fue realizado con humedades entre $4 \%$ y $25 \%$ para la arena calcárea y entre $2 \%$ y $8 \%$ para la arena de sílice. Las densidades relativas $\left(D_{r}\right)$ para todas las muestras variaron entre $20 \%$ y $75 \%$ al final de la preparación de la muestra.

Para saturar las muestras fue necesario circular un flujo de $\mathrm{CO}_{2}$ a través de las muestras por un periodo de 30 a 45 minutos. Este procedimiento ha sido realizado por otros autores (eg., Naeini \& Moayed, 2007), y su finalidad es reemplazar el aire atrapado dentro de la muestra por $\mathrm{CO}_{2}$, debido a que éste entra en solución con el agua a bajas presiones, facilitando el proceso de saturación. Las presiones de flujo utilizadas para circular el $\mathrm{CO}_{2}$ estuvieron entre $15 \mathrm{kPa}$ y $30 \mathrm{kPa}$. Después de este proceso, se procedió a pasar agua des-aireada, con el fin de expulsar el $\mathrm{CO}_{2}$ presente en los poros.

Este proceso se llevó a cabo hasta que visualmente no se notara la salida de $\mathrm{CO}_{2}$ de la muestra, y fluyera agua continuamente. Luego de colocar el flujo de $\mathrm{CO}_{2}$ y de circular agua des-aireada, se terminó el proceso de saturación convencional mediante la aplicación del método de contrapresión ("back pressure"). Este procedimiento fue llevado a cabo hasta obtener valores del parámetro B de Skempton (1954) mayores o iguales a 0.95 .

Terminado el proceso de saturación, los especímenes se consolidaron isotrópicamente bajo tres niveles de esfuerzos efectivos diferentes $(50,100$ y $200 \mathrm{kPa})$. Las densidades relativas de todas las muestras variaron entre $23 \%$ y $83 \%$ después de la etapa de consolidación.

Después de consolidar isotrópicamente las muestras, se esperó un tiempo de estabilización de
30 minutos antes de aplicar la carga cíclica. La fase cíclica se realizó bajo esfuerzo controlado sinusoidal, con una frecuencia de aplicación de la carga $(f)$ de $1 \mathrm{~Hz}$, sin permitir el drenaje de la muestra. La amplitud de la carga cíclica se eligió para obtener diferentes valores de esfuerzo cíclico normalizado (CSR). En la siguiente sección se presentan más detalles de la fase cíclica de los ensayos.

\section{Resultados}

En esta investigación, la resistencia a licuación fue presentada en términos de curvas de relación de esfuerzo cíclico (CSR), como una función del número de ciclos para producir licuación. En ensayos triaxiales cíclicos consolidados isotrópicamente, la relación de esfuerzo cíclico se define como la relación entre el esfuerzo cortante $(\tau)$ y el esfuerzo efectivo de consolidación isotrópica $\left(\sigma^{\prime}{ }_{3 c o n}\right)$. Debido a que en este tipo de ensayo triaxial, el esfuerzo cortante sobre el plano de interés es igual a la mitad del esfuerzo desviador cíclico aplicado $\left(\sigma_{\text {dcic }}\right)$, la CSR se expresa como se indica en la Ec. 1:

$$
\operatorname{CSR}=\frac{\tau}{\sigma_{3 \text { con }}^{\prime}}=\frac{\left(\sigma_{\text {dcic }}\right) / 2}{\sigma_{3 \text { con }}^{\prime}}
$$

Donde:

$$
\begin{aligned}
& \text { CSR: Relación de esfuerzo cíclico } \\
& \tau \text { : Esfuerzo cortante cíclico aplicado } \\
& \sigma_{\text {dcic }} \text { : Esfuerzo desviador cíclico aplicado } \\
& \sigma_{\text {3con }}^{\prime} \text { : Esfuerzo efectivo de consolidación } \\
& \text { isotrópico }
\end{aligned}
$$

Para cada densidad relativa y esfuerzo efectivo de consolidación, se realizaron entre tres y cinco ensayos triaxiales cíclicos para diferentes niveles de CSR. En cada prueba, se registró el número de ciclos necesario para producir licuación. El criterio de licuación se definió como una condición para la cual el esfuerzo efectivo es igual a cero (i.e., el exceso de presión de poros $(\Delta U)$ es igual al esfuerzo de consolidación), o cuando la doble amplitud de la deformación axial $(\varepsilon)$ fue de $5 \%$. Se prepararon curvas de resistencia a la 
licuación para cada densidad relativa y esfuerzo de consolidación, las cuales por tratarse de curvas de resistencia, suelen denominarse curvas de resistencia cíclica (CRR). La Figura 5 presenta las ocho curvas de resistencia cíclica obtenidas para la arena calcárea de Cabo Rojo. De manera similar, y utilizando las mismas escalas en los ejes, la Figura 6 ilustra las dos curvas de resistencia cíclica obtenidas para la arena silícea de Ottawa.

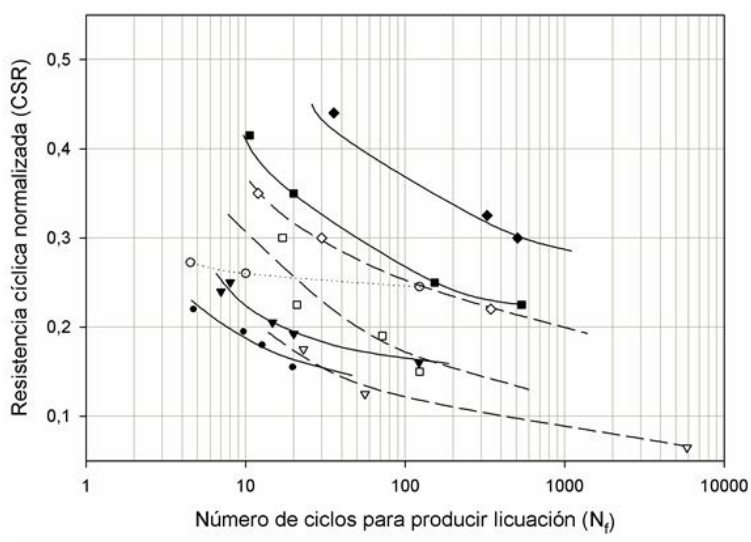

Figura 5. Curvas de resistencia cíclica (CRR) para todas las muestras de la arena calcárea de Cabo Rojo. $O \sigma_{3 c o n}^{\prime}=50 \mathrm{kPa}$ $D_{r}=26 \%,-\sigma_{3 \text { con }}^{\prime}=100 \mathrm{kPaD}=27 \%, \nabla \sigma^{\prime}{ }_{3 c o n}=100 \mathrm{kPaD}=$ $45 \%, \nabla \sigma_{3 c o n}^{\prime}=200 \mathrm{kPa} D_{r}=50 \%, \square \sigma_{3 c o n}^{\prime}=100 \mathrm{kPa} D_{r}=$ $64 \%, \square \sigma_{3 c o n}^{\prime}=200 \mathrm{kPa} D_{r}=65 \%, \diamond \sigma_{3 c o n}^{\prime}=100 \mathrm{kPa} D_{r}=$ $80 \%, \diamond \sigma_{3 c o n}^{\prime}=200 \mathrm{kPaD}=82 \%$,

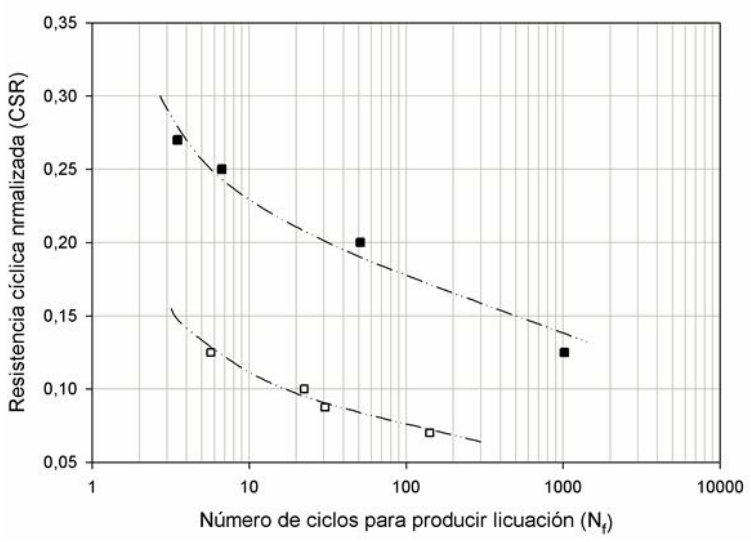

Figura 6. Curvas de resistencia cíclica (CRR) para todas las muestras de la arena silicea de Ottawa, $\square \sigma_{3 c o n}^{\prime}=50 \mathrm{kPa} D_{r}=$ $25 \%, \square \sigma_{3 c o n}^{\prime}=100 \mathrm{kPaD} D_{r}=68 \%$

\subsection{Principales factores que influyen en la resistencia a licuación}

Hay varios factores que influyen en la resistencia a la licuación de arenas. Por ejemplo, de la Figura 5 se pueden apreciar las familias de curvas de CRR desarrolladas para un mismo esfuerzo efectivo isotrópico de consolidación, donde la resistencia a licuación tiende a aumentar a medida que la densidad relativa de las muestras se incrementa. Esta observación hecha en las arenas calcáreas es consistente con resultados de investigaciones similares realizadas sobre arenas silíceas (e.g., Seed \& Lee, 1966).

Otro factor importante que influye en la resistencia a licuación es el nivel de consolidación isotrópico efectivo en las muestras antes de aplicar la carga cíclica no drenada. La Figura 7 ilustra curvas CRR para la arena de Cabo Rojo en un estado medio denso $\left(D_{\mathrm{r}}=64 \%-65 \%\right)$, consolidadas bajo esfuerzos efectivos de 100 y $200 \mathrm{kPa}$. En la Figura 7 se puede observar que para muestras probadas bajo densidades relativas similares, la resistencia a licuación CRR decrece cuando se incrementa el esfuerzo efectivo de consolidación. Cabe anotar que la magnitud de los esfuerzos cortantes cíclicos aplicados $\left(\tau_{\text {cic }}\right)$, es decir CRR multiplicado por $\sigma_{3 c o n}^{\prime}$, aumenta con el incremento del nivel de esfuerzo isotrópico efectivo de consolidación utilizado.

Sin embargo, es deseable mejor estudiar la resistencia a licuación dentro del marco de la mecánica de estado crítico, es decir en función de la posición de las muestras con respecto a la LEC justo al final de la fase de consolidación isotrópica y antes de la aplicación de la carga cíclica no drenada. Las posiciones iniciales con respecto a la LEC de los ensayos realizados en las arenas calcáreas se presentaron en la Figura 4. Como se mencionó antes, muestras por encima (o a la derecha) de la línea LEC suelen referirse como contráctiles, mientras que muestras por debajo (o a la izquierda) se denominan dilatantes. Una manera de representar esta posición relativa es mediante el parámetro de estado $(\Psi)$ (Been \& Jefferies, 1985). El parámetro de estado $(\Psi)$ involucra tanto la densidad relativa como el esfuerzo efectivo principal promedio, y se define como la diferencia 
entre la relación de vacíos de la muestra (e) y la relación de vacíos en estado crítico ( $\mathrm{e}_{\text {crit }}$ ) para el mismo esfuerzo efectivo. Entre más grande sea el valor algebraico de $\Psi$, mayor es la susceptibilidad a licuación. Muestras contráctiles tendrán un valor del parámetro de estado $\Psi$ positivo, mientras que muestras en el lado dilatante tendrán un valor de $\Psi$ negativo. Un ejemplo de la influencia del valor de la posición relativa a la línea LEC (usando $\Psi$ ) se presenta en la Figura 7. En esta figura se puede observar que la resistencia normalizada a licuación de las muestras consolidadas bajo un esfuerzo efectivo de $100 \mathrm{kPa}$ (lado dilatante, $\Psi=$ 0.017 ) es mayor que la resistencia obtenida para las muestras consolidadas bajo un esfuerzo efectivo de $200 \mathrm{kPa}$ (lado contráctil, $\Psi=+0.307$ ).

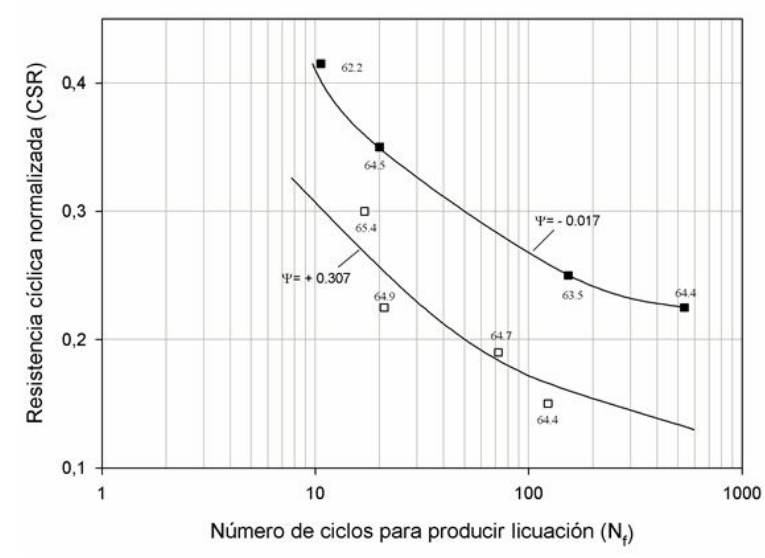

Figura 7. Efecto del nivel de esfuerzo efectivo de consolidación y del parámetro $\Psi$ en la resistencia a licuación de las arenas calcáreas de Cabo Rojo (estado medio denso, $\left.D_{r}=64-65 \%\right) . \quad \square \sigma_{3 c o n}^{\prime}=100 \mathrm{kPa} D_{r}=64 \%, \square \sigma_{3 c o n}^{\prime}=200$ $k P a D_{r}=65 \%$

\subsection{Comparación de curvas CRR para la arena de Cabo Rojo y la arena Ottawa}

La Figura 8 muestra curvas de resistencia cíclica de las dos arenas estudiadas. Estas curvas corresponden a muestras preparadas en estado suelto $\left(D_{\mathrm{r}}=23 \%-27 \%\right)$ y consolidadas isotrópicamente bajo un esfuerzo efectivo de 50 $\mathrm{kPa}$. La Figura 9 presenta una comparación similar, para muestras preparadas en estado medio denso $\left(\mathrm{D}_{\mathrm{r}}=64 \%-68 \%\right)$ y consolidadas bajo un esfuerzo efectivo de $100 \mathrm{kPa}$. En las Figuras 8 y 9 se puede observar que las arenas calcáreas de Cabo
Rojo presentaron una resistencia a licuación considerablemente mayor que la de las arenas de Ottawa, para muestras probadas en densidades relativas similares, bajo el mismo esfuerzo efectivo de consolidación. Estos resultados concuerdan con resultados reportados para arenas calcáreas de otras regiones del mundo, como arenas de Hawái según Ross \& Nicholson (1995) y Morioka \& Nicholson(2000).

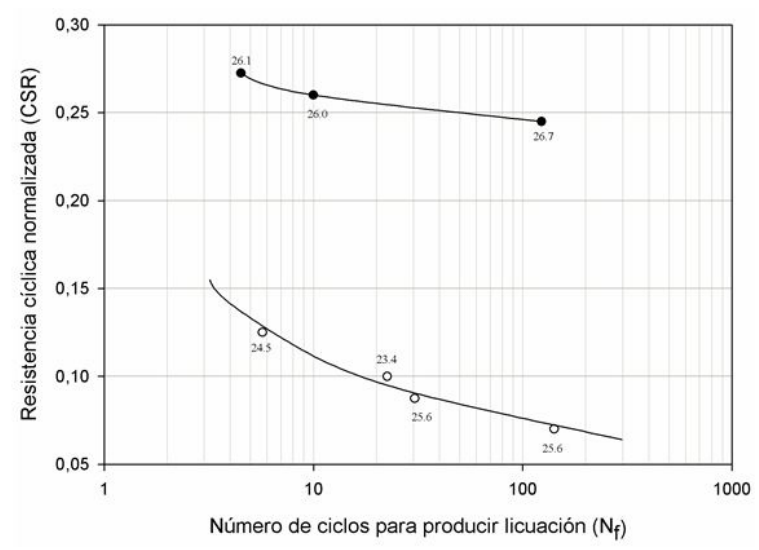

Figura 8. Comparación de curvas CRR para la arena calcárea y la arena de sílice (estado suelto, $\sigma^{\prime}{ }_{3 c o n}=50 \mathrm{kPa}$ ).

- Arena Cabo Rojo, O Arena Ottawa

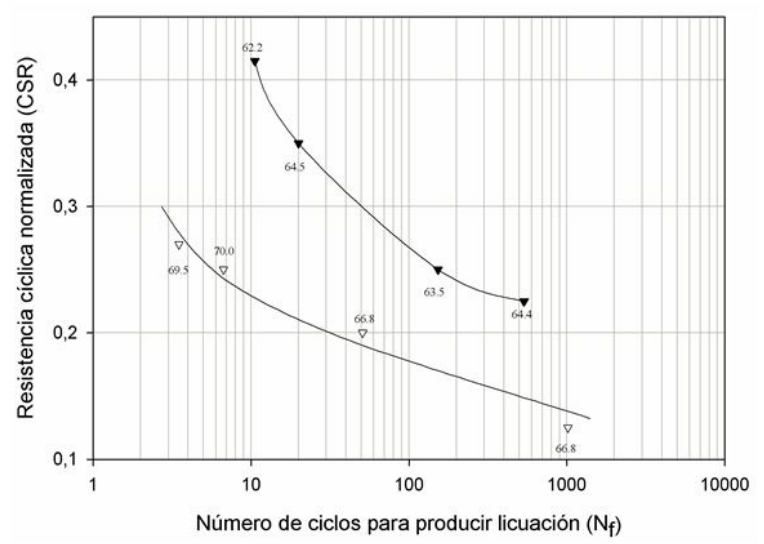

Figura 9. Comparación de curvas CRR para la arena calcárea y la arena de sílice (estado medio denso, $\sigma^{\prime}{ }_{3 c o n}=100$ kPa). $\nabla$ Arena Cabo Rojo, $\nabla$ Arena Ottawa

\subsection{Comparación de curvas de generación de excesos de presión de poros}

Durante la fase cíclica, debido a que no se permite el drenaje de la muestra, se generan excesos de 
presión de poros, que pueden llegar a igualar el esfuerzo efectivo de consolidación con el que inicia la fase cíclica, momento en el cual ocurre la licuación. La Figura 10 ilustra curvas de generación de excesos de presión de poros para las dos arenas estudiadas, para muestras preparadas en estado suelto $\left(D_{r}=25 \%-26 \%\right)$, y consolidadas isotrópicamente bajo un esfuerzo efectivo de 50 $\mathrm{kPa}$. Una comparación similar se presenta en la Figura 11, para muestras en estado medio-denso $\left(D_{r}=64 \%-68 \%\right)$, consolidadas bajo un esfuerzo efectivo de $100 \mathrm{kPa}$. El eje de las ordenadas en estas dos figuras, corresponde al exceso de presión de poros residual $\left(\Delta \mathrm{U}_{\text {res }}\right)$, normalizado respecto al esfuerzo efectivo de consolidación $\left(\sigma^{\prime}{ }_{\text {zcon }}\right)$. El exceso de presión de poros residual $\left(\Delta \mathrm{U}_{\text {res }}\right)$ se define como el valor del exceso de presión de poros cuando el esfuerzo desviador aplicado $\left(\sigma_{\mathrm{d}}\right)$ es igual a cero durante cada ciclo de carga (Seed \& Lee, 1966). El eje de las abscisas indica el número de ciclos en cada instante de la prueba, normalizado respecto al número de ciclos requeridos para producir licuación (i.e., $\mathrm{N} / \mathrm{N}_{\mathrm{f}}$ ).

En las Figuras 10 y 11 se pueden apreciar importantes diferencias en cuanto a las características de generación de excesos de presión de poros de las arenas calcáreas comparadas con las arenas de sílice. Por ejemplo, cerca al inicio de la fase de aplicación de la carga cíclica, la arena calcárea desarrolló valores más altos de excesos de presión de poros que la arena de sílice, cuando fueron ensayadas bajo el mismo esfuerzo de consolidación y densidades relativas similares. Otra diferencia importante es que la arena calcárea presentó fluctuaciones marcadas en los excesos de presión de poros entre cargas cíclicas, indicando una especie de relajación entre ciclos de carga; mientras que la arena de sílice mostró un lento y gradual exceso de presión de poros al inicio de la fase cíclica, y no se observaron fluctuaciones importantes entre ciclos de carga. Como tercera diferencia, al final de la prueba, cuando la muestra se aproxima a licuación, las arenas de sílice típicamente mostraron un incremento súbito y repentino en los excesos de presión de poros, mientras que las arenas calcáreas presentaron un incremento lento y gradual hasta llegar a licuación, menor que el incremento presentado al inicio de la fase cíclica.

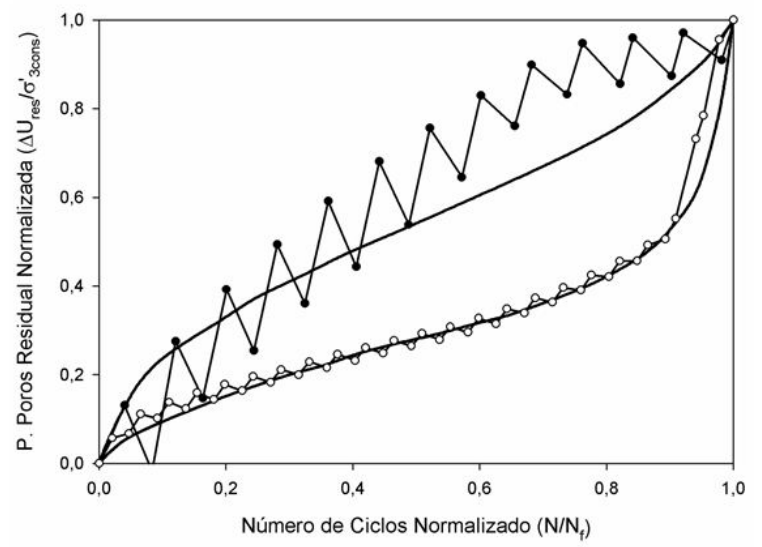

Figura 10. Comparación de curvas de presión de poros para las arenas ensayadas (estado suelto, $\sigma_{3 \text { con }}^{\prime}=50 \mathrm{kPa}$ ). - Arena Cabo Rojo, OArena Ottawa, - Límite Seed et al., (1975)

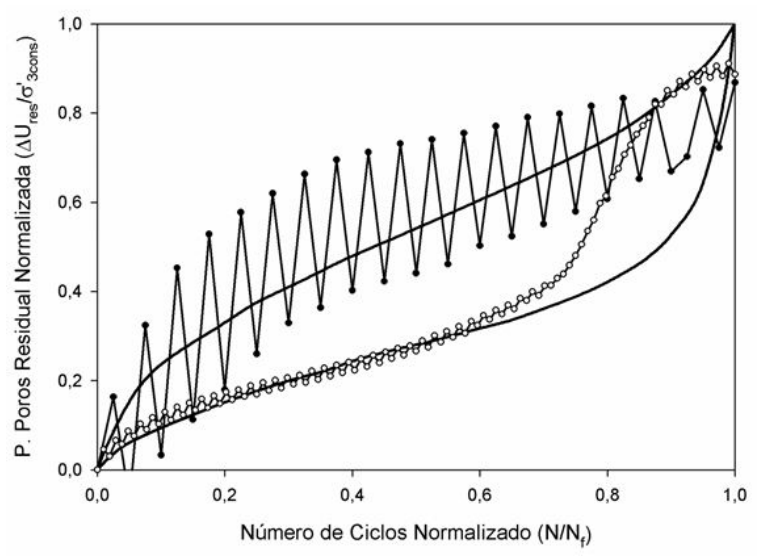

Figura 11. Comparación de curvas de presión de poros para las arenas ensayadas (estado medio-denso, $\sigma^{\prime}{ }_{3 c o n}=100 \mathrm{kPa}$ ). - Arena Cabo Rojo, O Arena Ottawa, -Límite Seed et al., (1975)

Las grandes fluctuaciones en los excesos de presión de poros durante la fase cíclica, presentadas por las arenas calcáreas, podrían ser atribuidas a un reacomodo de las partículas, debido a sus características específicas como granos con formas únicas, rugosidad superficial, porosidad interna, entre otras. Esta relajación en los excesos de presión de poros, podría contribuir a la alta resistencia a licuación presentada por las arenas calcáreas comparada con la de las arenas de sílice. Las características en la generación de excesos de presión de poros de las arenas calcáreas de Cabo Rojo fueron similares a lo reportado por 
Ross \& Nicholson (1995) y por Morioka \& Nicholson (2000) para dos arenas calcáreas de Hawái.

En las Figuras 10 y 11 se muestra también un rango típico de generación de excesos de presión de poros reportado por Seed et al., (1975). El rango propuesto se basó en resultados de laboratorio obtenidos por Lee \& Albaisa (1974), quienes realizaron ensayos triaxiales cíclicos sobre muestras reconstituidas de seis tipos de arenas de sílice de grano uniforme. En estas figuras se puede observar que las arenas de sílice de Ottawa se ajustan bien al límite inferior de la banda sugerida por Seed et al., (1975). En contraste, las arenas calcáreas tienden a estar más cerca del límite superior del rango indicado; sin embargo, debido a la alta relajación en las presiones de poros, se salen en varios puntos de la banda superior. Esta tendencia es más evidente cuando se incrementa la densidad relativa de la muestra.

\subsection{Comparación en la acumulación de deformaciones axiales}

$\mathrm{Al}$ acercarse al estado de licuación, la muestra en un ensayo triaxial cíclico experimenta un incremento marcado en las deformaciones axiales. Esto se debe a que el suelo pierde gran parte o toda su rigidez al alcanzar el estado de licuación, que se caracteriza por tener un esfuerzo efectivo de cero o muy cercano a cero. La manera como se acumulan las deformaciones axiales en la muestra hasta llegar a la licuación depende en gran medida de la posición inicial de la muestra con respecto a la línea de estado crítico, es decir de si su estado inicial antes del corte es contráctil o dilatante. Otro factor importante que influye en la forma cómo se acumulan las deformaciones axiales, son las características individuales de los granos. Al igual que para las presiones de poros, las propiedades tan singulares que tienen las partículas de las arenas calcáreas tienen gran influencia en la forma cómo se acumulan las deformaciones axiales en estos suelos durante ensayos triaxiales cíclicos.

La Figura 12 ilustra simultáneamente la acumulación de deformación axial de las arenas calcáreas y de sílice, para muestras en estado suelto $\left(D_{r}=25 \%-26 \%\right)$ consolidadas bajo un esfuerzo efectivo de $50 \mathrm{kPa}$. Una comparación similar se presenta en laFigura 13 para muestras en estado medio denso $\left(\mathrm{D}_{\mathrm{r}}=64 \%-68 \%\right)$ consolidadas bajo un esfuerzo efectivo de $100 \mathrm{kPa}$.

En las Figuras 12 y 13 puede notarse que las arenas calcáreas experimentan un incremento en la amplitud de la deformación axial desde el inicio de la carga cíclica, el cual es gradual hasta el momento de la licuación. Estas arenas no presentan crecimiento drástico en la deformación axial, aún al llegar al estado de licuación. Por otra parte, las arenas de sílice se deforman muy poco a lo largo de la prueba cíclica, pero experimentan un

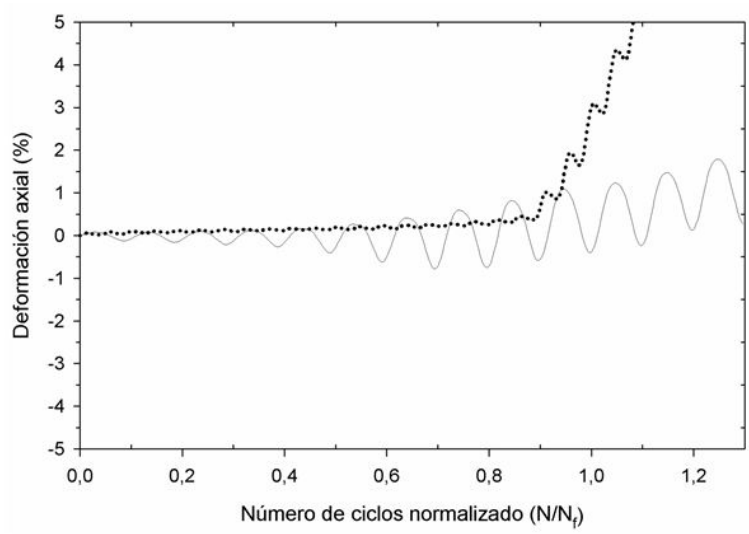

Figura 12. Comparación de curvas de deformación axial para las arenas ensayadas (estado suelto, $\sigma^{\prime}{ }_{3 c o n}=50 \mathrm{kPa}$ ). -Arena Cabo Rojo, ---Arena Ottawa

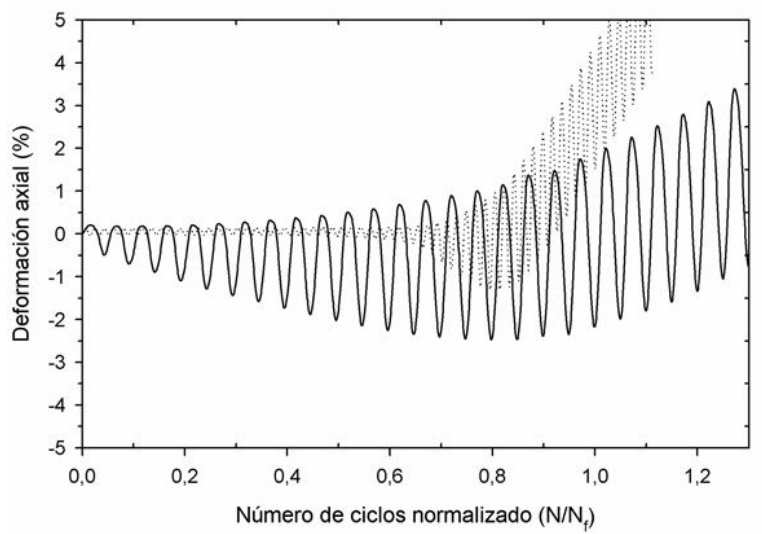

Figura 13. Comparación de curvas de deformación axial para las arenas ensayadas (estado medio-denso, $\sigma^{\prime}{ }_{3 c o n}=100$ kPa).-Arena Cabo Rojo, ---Arena Ottawa 
incremento súbito en la deformación axial un poco antes de la licuación, justo cuando se incrementan las presiones de poros. La amplitud de la deformación axial para ambas arenas, se incrementa con el aumento de la densidad relativa.

\section{Conclusiones}

Los resultados obtenidos mostraron que las arenas calcáreas de Cabo Rojo tienen mayor resistencia a la licuación que las arenas de sílice de Ottawa. Este comportamiento se evidenció en curvas de resistencia cíclica más altas para las arenas calcáreas con respecto a las de las arenas de sílice, para muestras ensayadas bajo condiciones similares.

Comparaciones realizadas entre la generación de excesos de presión de poros de las dos arenas estudiadas, indicaron que las arenas calcáreas tienen características diferentes a la tendencia general de generación de excesos de presión de poros. Las arenas calcáreas tuvieron picos iniciales más altos, sin embargo, el crecimiento de la presión de poros fue gradual desde el inicio hasta alcanzar el estado de licuación. Por otra parte, aunque las arenas de sílice no presentaron picos iniciales de presiones de poros tan altos, experimentaron un crecimiento súbito al acercarse al estado de licuación.

Las arenas calcáreas tuvieron un incremento de la deformación axial desde el inicio de la carga cíclica, el cual es gradual hasta el estado de licuación, sin presentar crecimientos drásticos. Las arenas de sílice por otra parte, se deformaron muy poco a lo largo de la prueba cíclica, pero sufrieron un incremento súbito en la deformación axial un poco antes del estado de licuación.

La forma y alta rugosidad de los granos de las arenas calcáreas puede generar mayor fricción entre partículas, y podría ser la responsable de que la generación de presiones de poros y resistencia a licuación de las arenas calcáreas sean considerablemente diferentes comparadas con las arenas de sílice. La alta resistencia cíclica de las arenas calcáreas también podría ser debido a que se presenten deformaciones elásticas individuales de las partículas, las cuales se recuperan durante la fase de extensión de la carga cíclica.

\section{Agradecimientos}

Los autores agradecen grandemente el apoyo financiero recibido por parte del Programa de Movimiento Fuerte de Puerto Rico, el Programa Sea Grant de Puerto Rico y el Programa NEHRP del USGS de los Estados Unidos, los cuales ayudaron a financiar parcialmente este estudio.

\section{Nomenclatura}

B: Parámetro de presión de poros de Skempton (adimensional)

$\mathrm{C}_{\mathrm{c}}$ : Coeficiente de gradación o curvatura (adimensional)

CRR: Relación de resistencia cíclica (adimensional)

CSR: Relación de esfuerzo cíclico (adimensional)

$\mathrm{C}_{\mathrm{u}}$ : Coeficiente de uniformidad (adimensional)

$\mathrm{D}_{10}$ : Diámetro efectivo de partículas $(\mathrm{mm})$

$\mathrm{D}_{50}$ : Diámetro promedio de partículas $(\mathrm{mm})$

$\mathrm{D}_{\mathrm{r}}$ : Densidad relativa $(\%)$

$\Delta \mathrm{U}:$ Exceso de presión de poros $(\mathrm{kPa})$

$\Delta \mathrm{u}_{\mathrm{res}}$ : Exceso de presión de poros residual $(\mathrm{kPa})$

e: Relación de vacíos

$\mathrm{e}_{\text {crit }}:$ Relación de vacíos para el estado crítico

$\mathrm{e}_{\max }:$ Relación de vacíos máxima (adimensional)

$\mathrm{e}_{\min }$ : Relación de vacíos mínima (adimensional)

$\varepsilon:$ Deformación unitaria axial (\%)

$\phi^{\prime}$ : Ángulo de fricción interna efectivo

f: Frecuencia de aplicación de la carga cíclica $(\mathrm{Hz})$

$\mathrm{G}_{\mathrm{s}}$ : Gravedad específica de los sólidos (adimensional)

$\gamma_{\max }:$ Peso unitario máximo $\left(\mathrm{kN} / \mathrm{m}^{3}\right)$

$\gamma_{\min }:$ Peso unitario mínimo $\left(\mathrm{kN} / \mathrm{m}^{3}\right)$ 
$\mathrm{p}^{\prime}$ : Esfuerzo efectivo principal promedio

$\sigma_{3}^{\prime}:$ Esfuerzo efectivo principal menor $(\mathrm{kPa})$

$\sigma_{3 \text { con }}^{\prime}$ : Esfuerzo efectivo principal menor de consolidación $(\mathrm{kPa})$

$\sigma_{\mathrm{d}}:$ Esfuerzo desviador $(\mathrm{kPa})$

$\tau$ : Esfuerzo cortante $(\mathrm{kPa})$

$\tau_{\text {cic }}:$ Esfuerzo cortante cíclico $(\mathrm{kPa})$

$\Psi:$ Parámetro de estado (adimensional)

\section{Referencias bibliográficas}

Been K., \& Jefferies M.G. (1985). A state parameter for sands. Geotechnique 35(2), 99-112.

Carraro, J. A. H., Bandini, P., \& Salgado, R. (2003). Liquefaction resistance of clean and nonplastic silty sands based on cone penetration resistance. Journal of Geotechnical and Geoenvironmental Engineering 129(11), 965976.

Cataño, J., \& Pando, M.A. (2010). Static and dynamic properties of a calcareous sand from southwest Puerto Rico. In Proceedings of the Annual Geo-Congress of the Geo-Institute of ASCE, West Palm Beach, FL, USA, p. 842-851.

Ishihara, K. (1996). Soil behavior in earthquake geotechnics. Tokyo, Japan: Oxford Science Publications.

Ladd, R. S. (1978). Preparing test specimen using under-compaction. Geotechnical Testing Journal 1(1), 16-23.

Lee, K. L., \& Albaisa, A. (1974). Earthquake induced settlements in saturated sands. Journal of the Geotechnical Engineering Division 100(4), 387-406.

Morioka, B. T., \& Nicholson, P. G. (2000). Evaluation of the liquefaction potential of calcareous sand. In proceedings of the 10th International Offshore and Polar Engineering Conference, Seattle, WA, USA, p. 494-500.

Naeini, S.A., \& Moayed, R.Z. (2007). Evaluation of undrained shear strength of loose silty sands using CPT results. International Journal of Civil Engineering 5(2), 104-117.

Polito, C. P., \& Martin, J. R. (2001). Effects of nonplastic fines on the liquefaction resistance of sands. Journal of Geotechnical and Geoenvironmental Engineering 127(5), 408-415.

Robertson, P. K., \& Wride, C. E. (1997). Liquefaction of sands and its evaluation. In proceedings of the 1st International Conference on Earthquake Geotechnical Engineering, Tokyo, Japan, p. 1253-1289.

Ross, M. S., \& Nicholson, P. G. (1995). Liquefaction potential and cyclic loading response of calcareous soils. Research Report UHM/CE/95-05, University of Hawaii, Manoa, HI, USA.

Santamarina, J. C., \& Cho, G. C. (2001). Determination of critical state parameters in sandy soils - simple procedure. American Society for Testing and Materials, 24(2), 185-192.

Seed, H. B., \& Lee, K. L. (1966). Liquefaction of saturated sands during cyclic loading. Journal of the Soil Mechanics and Foundation Division 92(3), 25-58.

Seed, H. B., Martin, P. P., \& Lysmer, J. (1975). The generation and dissipation of pore water pressures during soil liquefaction. Earthquake Engineering Research Center Report No EERC 75-29, University of California, Berkeley, CA, USA.

Skempton, A. W. (1954). The pore pressure coefficients A and B. Geotechnique 4, 143-147. 